Ольга Гвоздяк

кандидат педагогічних наук, доцент, завідувачка кафедри німецької філології ДВНЗ «Ужггородський національний університет» ORCID https://orcid.org/0000-0002-5760-576X

Ужггород, Україна ,+38 0505504177 olha.hvozdyak@uzhnu.edu.ua

Тетяна Свида-Сусіденко ст. викладач кафедри німечької філології ДВНЗ «Ужггородський національний університет» ORCID 0000-0002-9210-5170 Ужггород, Украӥна,+38 0662522722 tetyana.svyda-susidenko@uzhnu.edu.ua

\title{
Übungen und Aufgaben zur Förderung der Schreibfertigkeiten im DaF-Unterricht
}

Анотація. У сучасній методиці навчання іноземною мовою виділяють чотири види мовленнєвої діяльності: говоріння, читання, аудіювання, письмо. Три перших види визначаються науковиями як об'єкти навчання. Письмо розглядається тільки як засіб оволодіння мовою.

Тому на практиці в навчальному процесі иьому виду мовленнєвої діяльності не завжди приділясться належна увага. Писемне мовлення може бути виражене, на відміну від усного мовлення, якому притаманні як лінгвістичні так $i$ екстралінгвістичні фактори, лише через лексикограматичні засоби. Багатолітній досвід роботи у виші дозволяе нам констатувати, що для якісного формування у студентів навиків письма необхідно вдосконалювати у них навички оформлення $i$ змістового наповнення писемних висловлювань шляхом якісного виконання різного роду вправ. Виділяємо різні рівні роботи з письмом: словникова робота (лексикограматичні вправи), робота з текстом, творче письмо. Навчання письму будується за принципом поступового зростання труднощів. У результаті опитування студентів 1 курсу відділення німецької філології ДВНЗ «УжНУ» було встановлено, що найважчим видом письмової роботи, на думку студентів є творче письмо, яке поєднує у собі комплекс знань лексики, граматичних норм, вміння логічно викласти думки тощо. Письмова мова служить надійним інструментом для розвитку мислення, стимулює говоріння, слухання і читання на іноземній мові.

Ключові слова: письмо, німецька мова, вправи, лексика, творче письмо 
Abstract. Modern methodology of foreign language teaching distinguishes four types of speech activities: speaking, reading, listening and writing. The first three types are defined by scholars as objects of learning. Writing is regarded as a means of language acquisition. That is why, in practice this type of activity is not given the proper attention in the process of teaching. Written speech can be expressed only through lexical-grammatical means as opposed to oral speech which can rely on both linguistic and extralinguistic factors. Our university experience of many years enables us to state that in order to form students' writing competence it is necessary to improve their skills of written speech by doing different kinds of exercises. There are various kinds of them: vocabulary work (lexical-grammatical exercises), work with the text and creative writing. Teaching writing is based on the principle of the gradual increase in difficulties. The result of the survey of the first year students of the German department of UzhNu shows that the most difficult kind of written work is creative writing, which comprises a set of language competencies ranging from vocabulary, grammar to the ability to set forth your ideas. Written speech allows us to preserve language and practical knowledge, serves as a reliable tool of critical thinking, and stimulates speaking, listening and reading in a foreign language.

Keywords: writing, German, exercises, vocabulary, work with text, creative writing

Einleitende Bemerkungen. Die Zeiten, in denen im Fremdsprachenunterricht nur die Grammatik, Vokabeln auswendig gelernt, die Texte gelesen und übersetzt wurden, sind vorbei. Das Bildungswesen in der Ukraine schenkt derzeit viel Bedeutung dem kommunikativen Ansatz im Fremdsprachenunterricht. Die Aufmerksamkeit wird dem Hören, Sprechen und Lesen geschenkt. Trotzdem wird das Schreiben im heutigen Fremdsprachenunterricht vernachlässigt. Das Schreiben wird oft von Wissenschaftlern und Hochschullehrern nur als Mittel zur Beherrschung dieser drei Fertigkeiten betrachtet.

Nach Auffassung von H.-J. Krumm, steht das Schreiben in dem kommunikativ orientierten DaF-Unterricht nicht im Vordergrund und dabei wird der Förderung der Schreibfertigkeit wenig Bedeutung beigemessen, denn der kommunikative Ansatz orientiert sich in erster Linie an der gesprochenen Sprache, d.h. das Schrieben wird im DaF-Unterricht meistens zugunsten der Förderung des Sprechens vernachlässigt [5, S. 23].

Für den Fremdsprachenunterricht ist Schreiben eine wichtige Kontaktmöglichkeit zu einer fremden Struktur [2, S. 181]. Das Schreiben fördert den Fremdsprachenerwerb. Diese Fertigkeit verhilft dazu, die geistigen Handlungen, schriftlich zu fixieren und zu strukturieren. Die Schreibfertigkeit dient heutzutage als Sprachmittel zur Überprüfung von Kenntnissen sowohl der Schüler als auch der Studenten. Deshalb ist die Frage nach der systematischen Förderung der Schreibfertigkeit aktuell. 
Zielsetzung und Hauptaufgaben. In dieser Abhandlung beschäftigen wir uns mit dem Schreiben, den Übungen und Aufgaben, die die Fertigkeit Schreiben im DaF-Unterricht fördern.

Es ist bekannt, dass die Sprechfertigkeiten - Hören, Sprechen, Lesen, Schreiben - eng miteinander verbunden sind. Das Schreiben ist ein längerer und langsamerer Prozess als Sprechen. Das Schreiben wirkt auf die anderen Fertigkeiten aus und deshalb unterstützt den Spracherwerb.

In der Methodik des Fremdsprachenunterrichts kommt Schreiben als Mittlertätigkeit und als Zieltätigkeit vor. Um Schreiben als Mittlertätigkeit handelt es sich bei allen übenden Schreibaktivitäten, z.B. schriftliche Lücken oder Umformübungen, Diktate: Skizzieren eines Dialogverlaufs zur Vorbereitung eines Rollenspiels usw. Schreiben als Zieltätigkeit liegt immer dann vor, wenn die Lernenden Texte in der geschriebenen Sprache produzieren - zu realen Schreibanlässen, z.B. persönliche Stellungnahme zu einem Thema oder auch zu simulierten Schreibanlässen [7, S. 249].

Der Gegenstand der vorliegenden Abhandlung ist das Schreiben als Zieltätigkeit im DaF-Unterricht. Darunter verstehen wir die Befähigung der Studenten Inhalte $\mathrm{zu}$ verknüpfen, Texte $\mathrm{zu}$ strukturieren, Informationen an Kommunikationspartner zu vermitteln, über Lebensereignisse zu berichten usw. Dabei bedenken die Lernenden sprachliche Mittel: Vokabeln, grammatische Strukturen, Orthographie. Man achtet auf die Textsorten. Im Schreiben können Gedanken und Gefühle ausgedrückt werden.

Problemforschung. Um Schreibfertigkeit schrittweise aufzubauen, braucht man entsprechende Übungen und Aufgaben. Als Erstes möchten wir einen Blick auf die Aufgaben und Übungen werfen, die den Prozess des Schreibens optimal zu gestalten verhelfen. Dafür sehen wir es als nötig, den Unterschied zwischen Aufgabe und Übung zu erläutern.

Ergebnisse. H. Funk und andere Autoren definieren Aufgaben folgender Weise: Aufgaben sind all jene sprachliche Aktivitäten, die einen „Sitz im Leben“ haben, d.h. die in dieser Form nicht nur im Kursraum stattfinden: Wie schreibe ich einen Lebenslauf? Wie berichte ich über Erlebnisse? In diesem Sinne bezeichnet der Begriff Aufgabe alles, was man mit Sprache macht, etwa um sich zu informieren und sich mit anderen Menschen auszutauschen. Aufgaben sind die kommunikativen Lernziele des Fremdsprachenunterrichts. Um sie zu lösen brauchen wir Wörter, sprachliche Regeln, die Kenntnis von Textsorten und interkulturellem Verhalten ebenso wie landeskundliche Kenntnisse und die Fähigkeit zu flüssigem Sprechen und Schreiben. In den Aufgaben werden eine Vielzahl von Kenntnissen und Fertigkeiten integriert. Komplexe Lernaufgaben, die in der Fachliteratur als Zielaufgaben bezeichnet werden, können durch einzelne Aufgaben vorbereitet werden [1, S. 11].

Übungen bereiten Aufgaben vor, indem sie Wortschatz, Aussprache, Strukturen oder einzelne Fertigkeiten gezielt trainieren. Sie zielen auf die konkrete Anwendung und möglichst rasche Verfügbarkeit des Geübten und seine freie 
Anwendung in Aufgaben ab. Damit ist das wichtigste Kriterium einer Übung benannt: sie muss für die Lernenden erkennbar auf eine Aufgabe vorbereiten. Sie muss in einen inhaltlichen Zusammenhang, den Kontext, eingebettet sein, der zu Sprachhandlungen, also zu Aufgaben, hinführt [1, S. 14].

H.-W. Huneke und W. Steinig fassen die Grundunterschiede zwischen der Aufgabe und der Übung übersichtlich in der folgenden Tabelle zusammen [3, S. 221]:

Tabelle 1.

\section{Unterschied zwischen der Übung und der Aufgabe}

\begin{tabular}{|l|l|}
\hline \multicolumn{1}{|c|}{ Übungen } & \multicolumn{1}{c|}{ Aufgaben } \\
\hline $\begin{array}{l}\text { Ziel: korrekte Sprachverwendung; } \\
\text { sprachbezogen }\end{array}$ & $\begin{array}{l}\text { Ziel: Gelingen von Mitteilung und } \\
\text { Verstehen bei der Kommunikation in } \\
\text { der Lerngruppe; mitteilungsbezogen }\end{array}$ \\
\hline vom Lehrer erstellt & im Unterrichtsprozess entstanden \\
\hline $\begin{array}{l}\text { ein vorgeplanter Lösungsweg, eine } \\
\text { richtige Lösung: fordert und fördert } \\
\begin{array}{l}\text { Orientierung an der (sprachlichen) } \\
\text { Norm }\end{array}\end{array}$ & $\begin{array}{l}\text { Lösungswege müssen von den Lernern } \\
\text { gefunden werden: mehrere Lösungen } \\
\text { möglich: fordern und fördern } \\
\text { Autonomie }\end{array}$ \\
\hline $\begin{array}{l}\text { orientieren den Einzelnen auf die } \\
\text { Übungsvorlage }\end{array}$ & $\begin{array}{l}\text { fordern und fördern Kooperation mit } \\
\text { anderen }\end{array}$ \\
\hline $\begin{array}{l}\text { haben dienende Funktion: } \\
\text { Erleichterung der Lösung von } \\
\text { Aufgaben }\end{array}$ & $\begin{array}{l}\text { sind dem Übungsgeschehen } \\
\text { übergeordnet: erfordern Übungen, um } \\
\text { leichter lösbar zu sein }\end{array}$ \\
\hline tendenziell: „Geschlossenheit“ & tendenziell: „Offenheit“ \\
\hline
\end{tabular}

Aus der Tabelle geht hervor, dass die Übungen eine gewisse Vorstufe der Verwendung einer Aufgabe darstellen. Während die Übungen nämlich dazu dienen, neues sprachliches Wissen und Können $\mathrm{zu}$ vervollkommnen, $\mathrm{zu}$ automatisieren und rasch verfügbar $\mathrm{zu}$ machen, damit es für die Alltagskommunikation vorhanden ist, und aufgrund dessen mit großem Maße mit der Steuerung und einer gewissen Geschlossenheit verbunden sind, werden die Aufgaben viel offener gestellt, womit sie unterschiedliche Möglichkeiten der Lösung leisten und damit auch größere Ansprüche auf die Autonomie des Lerners legen. Diesbezüglich ist jedoch zu bemerken, dass sich Übungen und Aufgaben nicht ausschließen, sondern bedingen und sich ergänzen. Die Übergänge dazwischen sind also ganz fließend und deswegen ist es nicht immer einfach, die Übung von der Aufgabe zu unterscheiden. Eine so genaue Kategorisierung ist jedoch auch nicht notwendig. Wesentlich ist es hauptsächlich, beide Formen sinnvoll und zweckmäßig im Unterricht anwenden zu können [3, S. 218-221]. 
Zur Förderung der Fertigkeit Schreiben bietet B. Kast eine Übungstypologie an. Dabei unterscheidet der Autor fünf Übungsbereiche: vorbereitende Übungen,

- $\quad$ aufbauende Übungen,

- $\quad$ strukturierende Übungen,

- $\quad$ freies, kreatives Schreiben,

- $\quad$ kommunikatives Schreiben [4, S. 34].

Als vorbereitende Übungen nennt man solche, die auf die Textproduktion vorbereiten. Das sind Übungen zur Aktivierung vorhandenen Wissens; Erarbeitung, Erweiterung und Festigung des Wortschatzes, wobei die Rechtschreibung zu beachten ist.

Wir setzen in unserem DaF-Unterricht solche Übungen und Spiele ein: Kofferpacken, Assoziationsspiel. Oft stehen verschiedene Variationen im Fokus, z.B. gleiche Wortarten, präpositionale Wendungen, Wortgruppen bzw. Redewendungen. Was die Sozialform betrifft, wird die Arbeit im Plenum durchgeführt. Bei fortgeschrittenen Studenten ist es ratsam Gruppen- oder Partnerarbeit einzusetzen.

Aufbauende Übungen haben zum Ziel zuerst die Sätze, danach Kleintexte zu bilden. Dabei werden geübte Wortschatz- und grammatische Strukturen gebraucht. Als Sozialform dominiert Einzelarbeit. Die Studenten bilden Sätze in entsprechender Zeitform, mit bestimmter Wortfolge und variierter Art des Subjekts. Danach werden einfache Sätze in Satzreihe oder Satzgefüge verwandelt, wobei der Gebrauch von Konnektoren zu beachten ist. Die Einzelsätze werden inhaltlich in Kleintexte verbunden.

Strukturierende Übungen steuern Textproduktion. Es geht um gesteuerte Arbeit am Text, bei derer Lösung man schon einen gewissen Grad an Produktivität aufweisen muss. Dazu zählen die Übungen:

- $\quad$ Rekonstruktion von Dialogen zu den Erzähltexten;

- Textergänzungsaufgaben;

- Umwandeln von Berichten, Nachrichten bzw. Zeitungsartikeln in Erzähltexte;

- $\quad$ Perspektivenwechsel;

- Zusammenfassung schreiben;

- $\quad$ Versprachlichung von Bildergeschichten;

- $\quad$ Individueller Plan zum Text.

Übungen zum kreativen und freien Schreiben bedeuten: die Lernenden frei schreiben lassen. Kreatives Schreiben kann durch strukturierende sprachliche Vorgaben initiiert werden [4, S. 126].

Freies Schreiben definiert P. Portmann als "nicht-vorlagengebundenes Schreiben", "die primäre Art des Schreibens, diejenige Form des mitteilenden Schreibens, welches die meisten Leute benützen, wenn sie überhaupt schreiben [6, S. 476]. 
B. Kast hält fest, dass freies Schreiben und Kreativität gehören zusammen, Kreativität und Strukturierung schließen sich nicht aus [4, S. 127].

Mit dem kreativen Schreiben haben wir uns in der gleichnamigen Abhandlung beschäftigt. Es wurden verschiedene Methoden des kreativen Schreibens erläutert und einige Beispiele für den Einsatz im Deutschunterricht angeführt [8]. Hier werden von uns häufig gebrauchte Schreibtechtiken des kreativen Schreibens im 1. Studienjahr genannt:

- $\quad$ Elfchen (ein Gedicht mit elf Wörtern in einer vorgegebenen Struktur),

- $\quad$ Schneeballgedicht (ein Gedicht mit wachsenden Zeilen nach einer vorgegebenen Struktur),

- $\quad$ Rondell (ein Gedicht mit acht Zeilen, von denen sich einige nach einen vorgegebenen Schema wiederholen.

Als Schreibübung mit Wörtern oder Sätzen wird im ersten Studienjahr Akrostikon (Gedicht, bei dem die jeweils ersten Buchstaben der aufeinander folgenden Zeilen aneinandergereiht ein Wort oder einen Satz ergeben; besonders beliebt ist diese Gedichtform auf der Grundlage des eigenen Namens) empfehlenswert.

Aus unserer Erfahrung könnten wir feststellen, dass oben genannte Methoden schon von Anfang an im Unterricht eingesetzt werden kann. Mit wenig Wortschatz und Grammatik können die Studierenden ihr eigenes Schreibprodukt vorstellen.

Unter dem kommunikativen Schreiben versteht man Übungen, die auf reale Kommunikationssituationen beziehen:

- Postkarten,

- Lebenslauf,

- $\quad$ Briefe per Post bzw. E-Mails,

- $\quad$ Anzeigen.

Beim Schreiben von Postkarten und Briefen werden wichtige Komponente dieser Textsorte berücksichtigt: Absender, Empfänger, Adresse, Ort und Datum, Anrede, Gruß- und Schlussformel.

Es ist zu bemerken, dass die oben gegebene Übungstypologie nicht zum Ziel hatte, einen so komplexen Prozess wie das Schreiben, genau in einzelnen Gruppen einzuteilen und sie voneinander abzugrenzen, sondern v.a. in der Menge von Übungen ein System und eine Transparenz zu bilden.

Bei jeder Übung sind den Studierenden konkrete Anleitungen zu geben, genaue Zeit zum Schreiben und Überarbeiten anzusagen. Musterbeispiele und Anleitungen sollen Schreibdenken und -freude nicht begrenzen. Die Aufgaben sollten so gestellt werden, dass die Studenten sowohl ihre Fähigkeiten, ihr Können als auch ihre Vorstellungen nutzen können.

In Ergebnis der Befragung der Studenten des 1. Studienjahres der Abteilung für deutsche Philologie der Nationalen Universität Uschhorod, welche der vier Fertigkeiten (Sprechen, Lesen, Hören und Schreiben) ihnen besonders schwer fallen, haben sie die Fertigkeit Schreiben genannt. 
Von den Arten des Schreibens fällt den Studenten das kreative Schreiben besonders schwer, weil es in sich einen Komplex der Kenntnisse in Lexik, Grammatik und das Können die Gedanken logisch auszudrücken verkörpert.

Schlussfolgerungen und Forschungsperspektiven. Die Entwicklung der Schreibkompetenz ist ein gesteuerter Prozess, der sich erst dann effektiv vollzieht, wenn die Schriftsprache mit kommunikativen Zwecken gebraucht wird. Deshalb soll die Aufmerksamkeit den Interessen und Bedürfnissen der Studierenden geschenkt werden.

Die Perspektive der Forschung sehen wir in der Ausarbeitung von Übungen, die der Entwicklung des kreativen Schreibens bei den Studenten des 1. Studienjahres beitragen.

\section{LITERATUR}

1. Funk H., Kuhn, Ch., Skiba D., Spaniel-Weise D. Wicke R. E. Aufgaben, Übungen, Interaktion. München: Klett-Langenscheidt, 2014. 184 S.

2. Heyd, G. Aufbauwissen für den Fremdsprachenunterricht (DaF). Ein Arbeitsbuch. Kognition und Konstruktion. Tübingen: Narr, 1997. 218 S.

3. Huneke H.-W., Steinig H.-W. Deutsch als Fremdsprache: Eine Einführung. Berlin: Erich Schmidt Verlag. 2009. 306 S.

4. Kast B. Fertigkeit Schreiben. Berlin: Langenscheidt, 1999. 232 S.

5. Krumm H.-J. Kulturgeprägte Einflüsse auf das Schreiben und ihre möglichen Einflüssen für den Schreibunterricht Deutsch als Fremdsprache. Tütken, G., Neuf-Münkel, G. (Hrsg.). Schreiben im DaF-Unterricht an Hochschulen und Studienkollegs. Bd. 1: Forschungsergebnisse - Didaktische Konzeption Übungsformen. Regensburg: Fachverband Deutsch als Fremdsprache, 1993. S. 23-33.

6. Portmann P. Schreiben und Lernen. Grundlagen der fremdsprachlichen Schreibdidaktik. Tübingen: Max Niemeyer, 1991. 611 S.

7. Storch G. Deutsch als Fremdsprache - eine Didaktik. Paderborn, 2008. 364 S.

8. Гвоздяк О.M., Свида-Сусіденко Т.В. Kreatives Schreiben im Deutschunterricht. Germanistik in der Ukraine. Jahrheft 7'2012. Київ: Видавничий центр КНЛУ, 2012. С. 221-237. 